\title{
Risk factor analysis for involvement of resection margins in gastric and esophagogastric junction cancer: an Italian multicenter study
}

\author{
Massimiliano Bissolati ${ }^{1}\left(\right.$ Matteo Desio $^{1} \cdot$ Fausto Rosa $^{2} \cdot$ Stefano Rausei $^{3}$ • \\ Daniele Marrelli ${ }^{4}$ Gian Luca Baiocchi ${ }^{5}$ Giovanni De Manzoni ${ }^{6}$. \\ Damiano Chiari ${ }^{1}$ - Giovanni Guarneri ${ }^{1}$ - Fabio Pacelli ${ }^{2}$ Lorenzo De Franco ${ }^{4}$. \\ Sarah Molfino $^{5} \cdot$ Chiara Cipollari $^{6}$ - Elena Orsenigo ${ }^{1}$
}

Received: 13 July 2015/Accepted: 21 December 2015/Published online: 5 January 2016

(c) The International Gastric Cancer Association and The Japanese Gastric Cancer Association 2016

\begin{abstract}
Background Resection margin (RM) involvement is associated with negative prognosis after gastrectomy. Intraoperative frozen section (IFS) analysis allows radical resection to be achieved in a single operation but is timeconsuming and resource-consuming. The aim of this study was to assess risk factors associated with RM involvement to identify patients who would benefit from IFS analysis. Methods We retrospectively analyzed patients who underwent gastrectomy with curative intent for gastric or esophagogastric junction (EGJ) cancer from 2000 to 2014 in six Italian hospitals. RM status was assessed by IFS analysis and/or definitive histopathology examination. A set of 21 potential risk factors were compared in a multivariate analysis between patients with positive RMs on IFS analysis or definitive histopathology examination and a control cohort of similar patients with negative RMs, with the samples stratified into three subgroups (T1, T2-T4
\end{abstract}

For the Italian Research Group for Gastric Cancer (G.I.R.C.G.).

Massimiliano Bissolati

maxbisso@gmail.com

1 Department of Surgery, San Raffaele Scientific Institute, Via Olgettina 60, 20132 Milan, Italy

2 Digestive Surgery, A. Gemelli Hospital, Catholic University, Rome, Italy

3 Department of Surgical Sciences and Human Morphology, First Division of Surgery, Insubria University, Varese, Italy

4 Surgical Oncology Unit, University of Siena, Siena, Italy

5 Surgical Clinic, Department of Experimental and Clinical Sciences, University of Brescia, Brescia, Italy

6 Department of Surgery, General and Upper G.I. Surgery Division, University of Verona, Verona, Italy
Lauren intestinal pattern, T2-T4 Lauren diffuse/mixed pattern).

Results One hundred forty-five patients had positive RMs. Survival was significantly worse in positive RM patients than in negative RM patients (89.5 months vs 28.9 months). Multivariate analysis showed that in $\mathrm{T} 1$ cancers a margin distance of less than $2 \mathrm{~cm}$ is a risk factor for RM involvement (odds ratio 15.7), in T2-T4 intestinal pattern cancers, serosa invasion (odds ratio 6.0), EGJ location (odds ratio 4.1), and a margin distance of less than $3 \mathrm{~cm}$ (odds ratio 4.0) are independent risk factors, and in T2-T4 diffuse/mixed pattern cancers, lymphatic infiltration (odds ratio 4.2), tumor diameter greater than $4 \mathrm{~cm}$ (odds ratio 3.5), EGJ location (odds ratio 2.8), and serosa invasion (odds ratio 2.2) are independent risk factors.

Conclusions Survival after gastrectomy is negatively affected by positive RMs. IFS analysis should be routinely used in patients with a high risk of positive RMs, especially in diffuse pattern cancers.

Keywords Resection margins - Gastric cancer . Esophagogastric junction · Gastrectomy · Risk factors . Intraoperative frozen section

\section{Introduction}

Resection margin (RM) clearance is one of the most debated topics in gastric cancer surgery. Whereas the prognostic impact or RM involvement by the tumor is still to be defined, especially in specific cases such as early gastric cancer (EGC) or low-stage gastric cancer [1-3], it is commonly accepted that positive margins are an independent unfavorable factor associated with worse survival after surgical resection $[4,5]$. Therefore, in the field of 
gastric cancer surgical oncology, every effort must be made to obtain a complete resection with negative margins and adequate lymph node dissection, which constitutes the only possible curative treatment for nonmetastatic gastric cancer, and reoperation should always be considered in the case of $\mathrm{R} 1$ resections [4].

The safe distance between the tumor and resection line that allows one to have a good chance of performing R0 surgery has been investigated in several studies [6-9], and the Japanese Gastric Cancer Association (JGCA) treatment guidelines [10] have established the minimal margin distance that should be observed, depending on the tumor's $\mathrm{T}$ category and growth pattern.

However, cases of resection line involvement even with apparently adequate distance are still possible, so the safest way to obtain negative RMs is to perform intraoperative frozen section (IFS) analysis, which has high specificity and sensitivity $[11,12]$ and allows one to determine the surgical extent of resection in the case of an infiltrated margin so as to achieve single-time radical surgery. Unfortunately, IFS analysis is extremely time-consuming and resource-consuming [13], and despite what some authors suggest $[14,15]$, its routine use in every case is not feasible.

The aim of this study was therefore to identify specific independent risk factors associated with proximal or distal RM involvement so as to identify those patients who would certainly benefit from IFS analysis.

\section{Materials and methods}

We retrospectively analyzed case series from six Italian university hospitals with a high gastric cancer surgery volume (San Raffaele Hospital, Milan; Policlinico A. Gemelli, Rome; Ospedale di Circolo e Fondazione Macchi, Varese; Azienda Ospedaliera Universitaria Senese, Siena; Spedali Civili, Brescia; Ospedale Borgo Trento, Verona). All patients with histologically proven gastric or esophagogastric junction (EGJ) cancer who underwent total or subtotal gastrectomy with curative intent from January 2000 to December 2015 were considered for this study. Proximal or distal RM clearance was assessed by definitive histopathology examinations (DHEs) on a surgical specimen in all cases, and, when available, also by IFS analysis. DHE was conducted with standard hematoxylin-eosin staining after the specimens had been fixed with formalin, whereas IFS analysis was conducted with fast hematoxylin-eosin staining after the samples had been cryofixed at $-70{ }^{\circ} \mathrm{C}$ [16].

A negative RM (R0 resection) was defined as the complete absence of both macroscopic and microscopic tumor involvement at the resection line on histopathology assessments. A microscopically positive RM (R1 resection) was defined as the presence of viable singular tumor cells or cell aggregates at the line of resection on IFS analysis, when available, and on DHE, in the absence of visible tumor involvement of the resection line. A macroscopically positive RM (R2 resection) was defined as the presence of visible tumor involvement of the resection line, confirmed by histopathology examinations.

Only patients with positive R1 proximal or distal RMs assessed either on IFS analysis (regardless of DHE) or, when IFS analysis was not available, on DHE were included in this study (group A). Exclusion criteria were as follows: Siewert type I and type II EGJ cancer, metastatic disease, palliative resections, R2 proximal or distal RM resection, positive (R1 or R2) circumferential margin resections, and previous gastric surgery.

The study group was compared with a homologous control cohort (group B) with the same exclusion criteria who underwent curative total or subtotal gastrectomy during the same time span for gastric or Siewert type III EGJ cancer, with R0 RM resection assessed on DHE. Since the completeness of the data is essential for the multivariate analysis, to correctly identify the risk factors, only patients having no more than two missing values for the variables listed below were included in the control group.

For all patients, the following demographic, clinical, and pathological variables were recorded and inserted in a computerized database: age, sex, BMI, elective/emergent surgery, type of gastrectomy, multivisceral resections, neoadjuvant chemotherapy (NACT), distance between the tumor and the involved margin (or the nearest margin in the case of $\mathrm{R} 0$ resection) assessed by the pathologist on a fresh specimen, EGJ location, tumor diameter, tumor grade, $\mathrm{T}$ category, $\mathrm{N}$ category, Lauren pattern, signet ring cell histological appearance, presence of multifocal disease, presence of tumor invasion within vascular or lymphatic vessels, presence of vascular, lymphatic, and perineural infiltration, human epidermal growth factor receptor 2 (HER2) gene overexpression/amplification [17], Mandard's tumor regression grade (TRG) [18] (only for patients who underwent NACT), and overall survival (OS). A global comparison of actuarial OS was conducted between DHE R0 resection patients and DHE R1 resection patients with the Kaplan-Meier test.

After an overall comparison of the aforementioned variables between group $\mathrm{A}$ and group $\mathrm{B}$, patients were stratified into three subgroups according to the JGCA treatment guidelines for a safe RM distance [10]: T1 patients; T2-T4 Lauren intestinal pattern cancer patients; T2-T4 Lauren diffuse/mixed pattern cancer patients.

For each subgroup, a univariate analysis was conducted between group A and group B patients for the following variables: 
- T1 patients: age, sex, BMI, elective/emergent surgery, RM distance from tumor (less than $2 \mathrm{~cm}$ or $2 \mathrm{~cm}$ or greater), EGJ location, tumor diameter, tumor grade (G1-G2 or G3-G4), Lauren pattern, signet ring cell histological appearance, multifocal disease, presence of tumor invasion, vascular infiltration, lymphatic infiltration, perineural infiltration, lymph node status (NO or $\mathrm{N}+$ ), HER2 gene overexpression/amplification.

- T2-T4 intestinal pattern patients: age, sex, BMI, elective/emergent surgery, multivisceral resection, NACT, RM distance from tumor (less than $3 \mathrm{~cm}$ or $3 \mathrm{~cm}$ or greater), EGJ location, tumor diameter, tumor grade (G1-G2 or G3-G4), signet ring cell histological appearance, multifocal disease, presence of tumor invasion, vascular infiltration, lymphatic infiltration, perineural infiltration, lymph node status (N0 or $\mathrm{N}+$ ), serosa invasion, HER2 gene overexpression/amplification, TRG (1-3 or $4-5)$.

- T2-T4 diffuse/mixed pattern patients: age, sex, BMI, elective/emergent surgery, multivisceral resection, NACT, RM distance from tumor (less than $5 \mathrm{~cm}$ or $5 \mathrm{~cm}$ or greater), EGJ location, tumor diameter, tumor grade (G1-G2 or G3-G4), signet ring cell histological appearance, multifocal disease, presence of tumor invasion, vascular infiltration, lymphatic infiltration, perineural infiltration, lymph node status (N0 or $\mathrm{N}+$ ), serosa invasion, HER2 gene overexpression/amplification, TRG (1-3 or $4-5)$.

Categorical data are presented as the percent proportion; continuous data are presented as the mean \pm standard deviation. For incomplete data, a dummy variable classified as "missing" was included in the analysis to allow the inclusion of all available cases. To compare differences in categorical data between the two groups, Fisher's exact test or the $\chi^{2}$ test was used, as appropriate. The unpaired $t$ test was used to compare continuous data between two groups. All variables found to be statistically significant on the univariate analysis were subsequently included in a multivariate binary logistic regression model to determine independent risk factors associated with RM involvement.

For all analyses, a two-tailed $p$ value less than 0.05 was considered as significant. Analyses were performed with the SPSS for Microsoft Windows version 22.0 (SPSS, Chicago, IL, USA).

\section{Results}

From 2000 to 2014, we identified in our series 191 patients who underwent gastric surgery who had positive RMs on IFS analysis or on DHE. In 92 patients (48\%), the proximal margin was involved, in 78 patients $(41 \%)$ the distal margin was involved, and in 21 patients $(11 \%)$ both margins were involved.

Forty-six patients (24\%) were excluded from the initial series: 19 patients $(41 \%)$ because of palliative surgery or the presence of metastatic disease, 13 patients $(28 \%)$ because of macroscopic evidence of (R2) margin involvement, eight patients $(18 \%)$ because of a positive (R1 or R2) circumferential margin, and six patients (13\%) because of previous gastric surgery. The remaining 145 patients were included in group A.

In $45 \%$ of patients the proximal margin was involved, in $45 \%$ the distal margin was involved, and in $10 \%$ both margins were involved. Although there was a higher incidence of proximal margin infiltration in the initial series than in the definitive patient group (48\% vs $45 \%$ ), no statistical difference was found $(p=0.764)$. Margin involvement was due to direct infiltration by the tumor in $83 \%$ of cases, whereas in the remaining $17 \%$ of cases it was due to the presence of neoplastic embolization on the resection line.

In 113 patients, because IFS analysis was not performed, RM involvement was assessed directly by DHE; in none of these patients had a second-time radicalization surgical procedure been performed. In the remaining 32 patients, the RM involvement was assessed by IFS analysis, and the subsequent surgical extent (esophageal resection in 20 patients, total gastrectomy in seven patients, and duodenal resection in five patients) allowed R0 surgery to be performed in all patients.

Demographic, clinical, and histopathological characteristics of the 145 patients are reported in Table 1.

The study group was compared with a control group, consisting of 529 patients operated on during the same period, each having no more than two missing values for the variables studied. Overall missing data for the control group amounted to $3 \%$.

\section{Survival analysis}

Postoperative OS of the 113 patients with DHE R1 resection was compared with that of the 561 patients with DHE radical resection (Fig. 1). The mean estimated OS was 89.5 months [95\% confidence interval (CI) 81.9-97.0 months] for R0 resection patients and 28.9 months $(95 \%$ CI 18.4-39.4 months) for $\mathrm{R} 1$ resection patients $(p<0.001)$. The 5-year OS of R0 and R1 resection patients was 59 and $22 \%$, respectively $(p<0.001)$.

\section{Overall comparison between group A and group B patients}

The overall comparison of analyzed variables between the whole group of positive RM patients and the control group 
Table 1 Demographic, clinical, and histopathological characteristics of 145 patients with proximal or distal resection margin $(R M)$ involvement on intraoperative frozen section analysis or definitive histopathology examination

\begin{tabular}{|c|c|}
\hline Variable & Value \\
\hline Age & $65.0 \pm 14.1$ years \\
\hline \multicolumn{2}{|l|}{$\operatorname{Sex}$} \\
\hline Male & $57 \%$ \\
\hline Female & $43 \%$ \\
\hline BMI & $24.2 \pm 3.7 \mathrm{~kg} / \mathrm{m}^{2}$ \\
\hline \multicolumn{2}{|l|}{ Type of surgery } \\
\hline Elective & $93 \%$ \\
\hline Emergent & $7 \%$ \\
\hline \multicolumn{2}{|l|}{ Type of gastrectomy } \\
\hline Subtotal & $48 \%$ \\
\hline Total & $52 \%$ \\
\hline Multivisceral resections & $13 \%$ \\
\hline NACT & $10 \%$ \\
\hline Nearest RM distance from tumor & $1.5 \pm 1.7 \mathrm{~cm}$ \\
\hline EGJ location & $26 \%$ \\
\hline Tumor diameter & $5.6 \pm 3.3 \mathrm{~cm}$ \\
\hline \multicolumn{2}{|l|}{ Tumor grade } \\
\hline G1 & $2 \%$ \\
\hline $\mathrm{G} 2$ & $19 \%$ \\
\hline G3 & $77 \%$ \\
\hline G4 & $2 \%$ \\
\hline \multicolumn{2}{|l|}{$\mathrm{T}$ category } \\
\hline $\mathrm{T} 1$ & $8 \%$ \\
\hline $\mathrm{T} 2$ & $10 \%$ \\
\hline $\mathrm{T} 3$ & $25 \%$ \\
\hline $\mathrm{T} 4$ & $57 \%$ \\
\hline \multicolumn{2}{|l|}{$\mathrm{N}$ category } \\
\hline No & $21 \%$ \\
\hline N1 & $11 \%$ \\
\hline $\mathrm{N} 2$ & $16 \%$ \\
\hline N3 & $52 \%$ \\
\hline \multicolumn{2}{|l|}{ Lauren pattern } \\
\hline Intestinal & $35 \%$ \\
\hline Diffuse/mixed & $65 \%$ \\
\hline Signet ring cell histological appearance & $53 \%$ \\
\hline Multifocal disease & $3 \%$ \\
\hline Tumor invasion & $24 \%$ \\
\hline Vascular infiltration & $24 \%$ \\
\hline Lymphatic infiltration & $25 \%$ \\
\hline Perineural infiltration & $16 \%$ \\
\hline HER2 gene overexpession/amplification & $3 \%$ \\
\hline \multicolumn{2}{|l|}{ TRG (only for NACT) } \\
\hline $1-3$ & $67 \%$ \\
\hline $4-5$ & $33 \%$ \\
\hline
\end{tabular}

$E G J$ esophagogastric junction, HER2 human epidermal growth factor receptor 2, NACT neoadjuvant chemotherapy, TRG tumor regression grade
OVERALL SURVIVAL

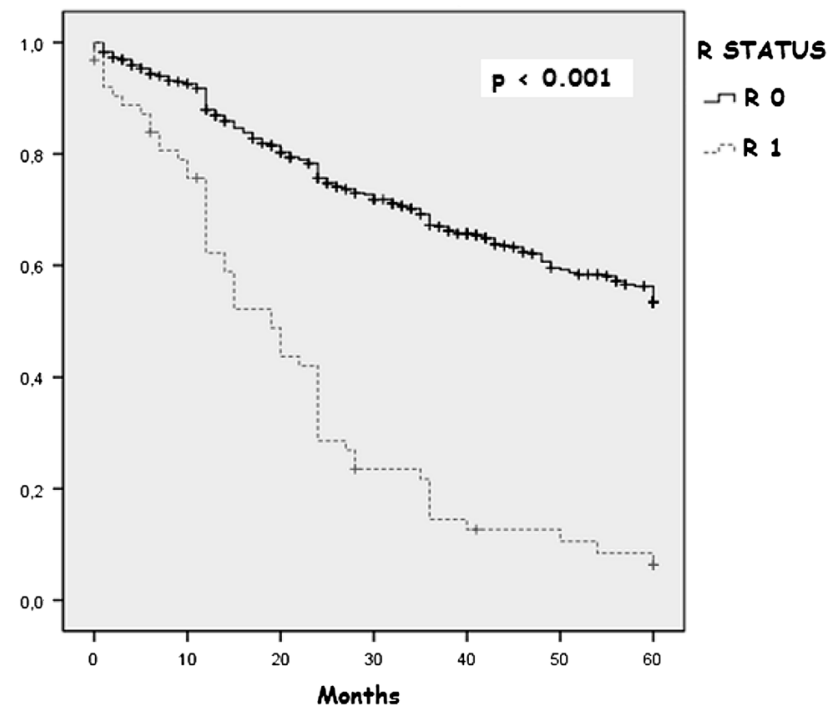

Fig. 1 Five-year overall survival of R0 and R1 resection patients

is reported in Table 2. Patients with RM involvement on IFS analysis or DHE had a higher incidence of EGJ location $(26 \%$ vs $12 \% ; p<0.001)$ and Lauren diffuse/mixed pattern cancer $(65 \%$ vs $36 \% ; p<0.001)$. In group A the tumor diameter was greater $(5.6 \pm 3.3 \mathrm{~cm}$ vs $3.9 \pm 2.4 \mathrm{~cm} ; p<0.001)$ and the margin distance was shorter $(1.5 \pm 1.7 \mathrm{~cm}$ vs $2.6 \pm 1.7 \mathrm{~cm} ; p<0.001)$ than in group B. The prevalence of T3-T4 cancer ( $82 \%$ vs $50 \%$; $p<0.001)$, N2-N3 cancer (68\% vs $40 \% ; p<0.001)$ and grade G3-G4 cancer (79 \% vs $59 \% ; p<0.001$ ) was significantly higher in positive RM patients. Moreover, group A patients had a higher incidence of signet ring cell histological appearance, tumor invasion, and vascular, lymphatic, and perineural infiltration (53\% vs $34 \%, 24 \%$ vs $8 \%, 24 \%$ vs $12 \%, 25 \%$ vs $7 \%$, and $16 \%$ vs $4 \%$, respectively; all $p<0.001)$. Finally, in patients with RM involvement, total gastrectomy (52\% vs $27 \%$; $p<0.001$ ) and multivisceral resections $(13 \%$ vs $2 \% ;<0.001)$ were more frequently performed.

\section{Risk factor analysis for $\mathrm{T} 1$ cancer patients}

Among the whole group, 175 patients had T1 cancer: 11 patients had RM involvement on IFS analysis or DHE (group A), whereas 164 had negative RMs (group B). Data regarding the comparison of potential risk factors between these two subgroups are reported in Table 3. Among all analyzed variables, only the RM distance from the tumor was significantly related with RM involvement on histological examination. In particular, there was an RM distance of less than $2 \mathrm{~cm}$ in $88 \%$ of group A patients but in 
Table 2 Comparison of demographic, clinical, and histopathological characteristics between 145 patients with resection margin $(R M)$ involvement on intraoperative frozen section analysis or definitive histopathology examination (group A) and 529 negative RM patients (group B)

\begin{tabular}{|c|c|c|c|}
\hline Variable & Group A (145 patients) & Group B (529 patients) & $p^{\mathrm{a}}$ \\
\hline Age & $65.0 \pm 14.1$ years & $66.9 \pm 12.0$ years & 0.131 \\
\hline \multicolumn{4}{|l|}{ Sex } \\
\hline Male & $57 \%$ & $57 \%$ & \multirow[t]{2}{*}{1.000} \\
\hline Female & $43 \%$ & $43 \%$ & \\
\hline BMI & $24.2 \pm 3.7 \mathrm{~kg} / \mathrm{m}^{2}$ & $24.9 \pm 4.3 \mathrm{~kg} / \mathrm{m}^{2}$ & 0.422 \\
\hline \multicolumn{4}{|l|}{ Type of surgery } \\
\hline Elective & $93 \%$ & $95 \%$ & \multirow[t]{2}{*}{0.252} \\
\hline Emergent & $7 \%$ & $5 \%$ & \\
\hline \multicolumn{4}{|l|}{ Type of gastrectomy } \\
\hline Subtotal & $48 \%$ & $73 \%$ & \multirow[t]{2}{*}{$<0.001$} \\
\hline Total & $52 \%$ & $27 \%$ & \\
\hline Multivisceral resections & $13 \%$ & $2 \%$ & $<0.001$ \\
\hline NACT & $10 \%$ & $14 \%$ & 0.303 \\
\hline Nearest RM distance from tumor & $1.5 \pm 1.7 \mathrm{~cm}$ & $2.6 \pm 1.7 \mathrm{~cm}$ & $<0.001$ \\
\hline EGJ location & $26 \%$ & $12 \%$ & $<0.001$ \\
\hline Tumor diameter & $5.6 \pm 3.3 \mathrm{~cm}$ & $3.9 \pm 2.4 \mathrm{~cm}$ & $<0.001$ \\
\hline \multicolumn{4}{|l|}{ Tumor grade } \\
\hline G1 & $2 \%$ & $12 \%$ & \multirow[t]{4}{*}{$<0.001$} \\
\hline G2 & $19 \%$ & $29 \%$ & \\
\hline G3 & $77 \%$ & $58 \%$ & \\
\hline G4 & $2 \%$ & $1 \%$ & \\
\hline \multicolumn{4}{|l|}{$\mathrm{T}$ category } \\
\hline $\mathrm{T} 1$ & $8 \%$ & $30 \%$ & \multirow[t]{4}{*}{$<0.001$} \\
\hline $\mathrm{T} 2$ & $10 \%$ & $20 \%$ & \\
\hline T3 & $25 \%$ & $33 \%$ & \\
\hline $\mathrm{T} 4$ & $57 \%$ & $17 \%$ & \\
\hline \multicolumn{4}{|l|}{$\mathrm{N}$ category } \\
\hline N0 & $21 \%$ & $45 \%$ & \multirow[t]{4}{*}{$<0.001$} \\
\hline N1 & $11 \%$ & $15 \%$ & \\
\hline $\mathrm{N} 2$ & $16 \%$ & $21 \%$ & \\
\hline N3 & $52 \%$ & $19 \%$ & \\
\hline \multicolumn{4}{|l|}{ Lauren pattern } \\
\hline Intestinal & $35 \%$ & $64 \%$ & \multirow[t]{2}{*}{$<0.001$} \\
\hline Diffuse/mixed & $65 \%$ & $36 \%$ & \\
\hline Signet ring cell histological appearance & $53 \%$ & $34 \%$ & $<0.001$ \\
\hline Multifocal disease & $3 \%$ & $3 \%$ & 1.000 \\
\hline Tumor invasion & $24 \%$ & $8 \%$ & $<0.001$ \\
\hline Vascular infiltration & $24 \%$ & $12 \%$ & $<0.001$ \\
\hline Lymphatic infiltration & $25 \%$ & $7 \%$ & $<0.001$ \\
\hline Perineural infiltration & $16 \%$ & $4 \%$ & $<0.001$ \\
\hline HER2 gene overexpession/amplification & $3 \%$ & $2 \%$ & 0.770 \\
\hline \multicolumn{4}{|l|}{ TRG (only for NACT) } \\
\hline $1-3$ & $67 \%$ & $42 \%$ & \multirow[t]{2}{*}{0.275} \\
\hline $4-5$ & $33 \%$ & $58 \%$ & \\
\hline
\end{tabular}

EGJ esophagogastric junction, HER2 human epidermal growth factor receptor 2, NACT neoadjuvant chemotherapy, TRG tumor regression grade

${ }^{a}$ Values in italic are significant 
Table 3 Univariate and multivariate analysis of potential risk factors for $\mathrm{T} 1$ cancers between patients with a positive proximal or distal resection margin $(R M)$ (group A) or a negative proximal or distal RM (group B)

\begin{tabular}{|c|c|c|c|c|c|c|}
\hline Variable & Group A (11 patients) & Group B (164 patients) & $p$ & OR & $95 \% \mathrm{CI}$ & $p$ \\
\hline Age & $67.3 \pm 8.6$ years & $65.2 \pm 11.7$ years & 0.569 & & & \\
\hline \multicolumn{7}{|l|}{ Sex } \\
\hline Male & $70 \%$ & $63 \%$ & 0.747 & & & \\
\hline Female & $30 \%$ & $37 \%$ & & & & \\
\hline BMI & $21.8 \pm 2.9 \mathrm{~kg} / \mathrm{m}^{2}$ & $24.9 \pm 4.4 \mathrm{~kg} / \mathrm{m}^{2}$ & 0.126 & & & \\
\hline \multicolumn{7}{|l|}{ Type of surgery } \\
\hline Elective & $100 \%$ & $98 \%$ & 1.000 & & & \\
\hline Emergent & $0 \%$ & $2 \%$ & & & & \\
\hline \multicolumn{7}{|c|}{ RM distance from tumor } \\
\hline$<2 \mathrm{~cm}$ & $88 \%$ & $31 \%$ & 0.002 & 15.7 & $1.9-131.9$ & $0.002^{\mathrm{a}}$ \\
\hline$\geq 2 \mathrm{~cm}$ & $12 \%$ & $69 \%$ & & & & \\
\hline \multicolumn{7}{|l|}{ EGJ location } \\
\hline No & $82 \%$ & $94 \%$ & 0.168 & & & \\
\hline Yes & $18 \%$ & $6 \%$ & & & & \\
\hline Tumor diameter & $3.4 \pm 2.5 \mathrm{~cm}$ & $2.6 \pm 1.7 \mathrm{~cm}$ & 0.159 & & & \\
\hline \multicolumn{7}{|l|}{ Tumor grade } \\
\hline G1-G2 & $73 \%$ & $53 \%$ & 0.348 & & & \\
\hline G3-G4 & $27 \%$ & $47 \%$ & & & & \\
\hline \multicolumn{7}{|l|}{ Lauren type } \\
\hline Intestinal & $54 \%$ & $66 \%$ & 0.514 & & & \\
\hline Diffuse/mixed & $46 \%$ & $34 \%$ & & & & \\
\hline \multicolumn{7}{|c|}{ Signet ring cell histological appearance } \\
\hline No & $70 \%$ & $64 \%$ & 1.000 & & & \\
\hline Yes & $30 \%$ & $36 \%$ & & & & \\
\hline \multicolumn{7}{|c|}{ Multifocal disease } \\
\hline No & $91 \%$ & $95 \%$ & 0.487 & & & \\
\hline Yes & $9 \%$ & $5 \%$ & & & & \\
\hline \multicolumn{7}{|l|}{ Tumor invasion } \\
\hline No & $90 \%$ & $96 \%$ & 0.353 & & & \\
\hline Yes & $10 \%$ & $4 \%$ & & & & \\
\hline \multicolumn{7}{|c|}{ Vascular infiltration } \\
\hline No & $91 \%$ & $95 \%$ & 0.461 & & & \\
\hline Yes & $9 \%$ & $5 \%$ & & & & \\
\hline \multicolumn{7}{|c|}{ Lymphatic infiltration } \\
\hline No & $91 \%$ & $98 \%$ & 0.237 & & & \\
\hline Yes & $9 \%$ & $1 \%$ & & & & \\
\hline \multicolumn{7}{|c|}{ Perineural infiltration } \\
\hline No & $91 \%$ & $100 \%$ & 0.070 & & & \\
\hline Yes & $9 \%$ & $0 \%$ & & & & \\
\hline \multicolumn{7}{|c|}{ Lymph node status } \\
\hline N0 & $27 \%$ & $22 \%$ & 0.711 & & & \\
\hline $\mathrm{N}+$ & $73 \%$ & $78 \%$ & & & & \\
\hline \multicolumn{7}{|c|}{ HER2 gene overexpression/amplification } \\
\hline No & $91 \%$ & $98 \%$ & 0.230 & & & \\
\hline Yes & $9 \%$ & $2 \%$ & & & & \\
\hline
\end{tabular}

CI confidence interval, EGJ esophagogastric junction, HER2 human epidermal growth factor receptor 2, $O R$ odds ratio

a Significant 
only $31 \%$ of group B patients $(p=0.002)$. The calculated OR for RM involvement in the case of an RM distance from the tumor of less than $2 \mathrm{~cm}$ was 15.7 (95\% CI $1.9-131.9 ; p=0.002)$. No other significant differences were found between the two groups concerning all the aforementioned variables.

When the analysis was restricted to only patients with gastric cancer, a free margin distance of less than $2 \mathrm{~cm}$ was still identified as the only risk factor (OR 12.3, $95 \% \mathrm{CI}$ 1.4-108.8, $p=0.002$ ).

\section{Risk factor analysis for T2-T4 Lauren intestinal pattern cancer patients}

Among the whole group, 274 patients had T2-T4 Lauren intestinal pattern cancer: 45 had RM involvement on IFS analysis or DHE (group A), whereas 229 had negative RMs (group B). Data regarding the comparison of potential risk factors between these two subgroups are reported in Table 4. In univariate analysis, multivisceral resections were more frequently performed in positive RM patients than in negative RM patients (18\% vs $2 \%$; $p<0.001)$. The distance between the tumor and the nearest RM was less than $3 \mathrm{~cm}$ in $91 \%$ of patients with RM involvement and in $63 \%$ of patients with R0 resection $(p=0.001)$. An ECJ location of the tumor was significantly more frequent in group A patients than in group B patients (36\% vs $20 \% ; p=0.03$ ). Grade G3 or G4 cancers were present in about $66 \%$ of positive RM patients but in only $49 \%$ of negative RM patients ( $p=0.048)$. The presence of tumor invasion, vascular infiltration, and lymphatic infiltration was significantly more frequent in group A patients $(24,33$, and $38 \%$, respectively) than in group $\mathrm{B}$ patients $(10 \%, p=0.024$; $16 \%, p=0.018$; and $10 \%, p=0.002$, respectively). Finally, patients with RM involvement had a significantly higher incidence of serosa invasion than patients with negative RMs (53\% vs $16 \%, p<0.001)$. No other significant differences in the distribution of the variables considered were found between the two subgroups on univariate analysis.

On multivariate analysis, a distance between the tumor and RM of less than $3 \mathrm{~cm}$ (OR 4.0, $95 \%$ CI 1.1-14.5, $p=0.03$ ), EGJ location (OR 4.1, $95 \%$ CI 1.7-10.0, $p=0.002$ ), and serosa invasion (OR 6.0, $95 \%$ CI $2.5-14.8, p<0.001$ ) were found to be the only independent risk factors for $\mathrm{RM}$ involvement by the tumor in patients with T2-T4 Lauren intestinal pattern cancer.

When the analysis was restricted to only patients with gastric cancer, serosa invasion (OR 6.7, $95 \%$ CI 2.1-21.4, $p=0.001$ ) and neoplastic embolization (OR 5.6, $95 \% \mathrm{CI}$ $1.4-22.5, p=0.016$ ) were the only independent risk factors identified.

\section{Risk factor analysis for T2-T4 Lauren diffuse/mixed pattern cancer patients}

Among the whole group, 225 patients had T2-T4 Lauren diffuse/mixed pattern cancer: 89 had RM involvement on IFS analysis or DHE (group A), whereas 136 had negative RMs (group B). Data regarding the comparison of potential risk factors between the two subgroups are reported in Table 5. On univariate analysis, $23 \%$ of RM-infiltrated patients had EGJ-located tumors, whereas only $8 \%$ of negative RM patients had tumors in this location $(p=0.003)$. Tumor diameter was significantly greater in group A patients than in group B patients $(6.1 \pm 3.7 \mathrm{~cm} \mathrm{vs}$ $4.1 \pm 2.1 \mathrm{~cm} ; p<0.001)$. Converting this continuous variable in a binary one, we find that $72 \%$ of group A patients had a tumor larger than $4 \mathrm{~cm}$ in comparison with $36 \%$ of group B patients $(p<0.001)$. Similarly to the previous analysis, also for this subgroup a significantly higher incidence of tumor invasion, vascular infiltration, and lymphatic infiltration was found in group A patients $(26,22$, and $26 \%$, respectively) in comparison with group $\mathrm{B}$ patients $(10 \%, p=0.002 ; 11 \%, p=0.037$; and $9 \%$, $p=0.001$, respectively). The presence of lymph node metastases was higher in patients with RM involvement than in patients with negative RMs (87\% vs $74 \%$, $p=0.03)$. Finally, serosa invasion was significantly more frequent in group A patients $(64 \%)$ than in group B patients $(37 \% ; p<0.001)$. No other significant differences in the distribution of the variables considered were found between the two subgroups on univariate analysis.

On multivariate analysis, EGJ location (OR 2.8, $95 \%$ CI 1.7-7.0, $p=0.033)$, tumor diameter greater than $4 \mathrm{~cm}$ (OR 3.5, $95 \%$ CI 1.8-6.7, $p<0.001$ ), presence of lymphatic infiltration (OR 4.2, $95 \%$ CI 1.7-10.3, $p=0.002$ ), and serosa invasion (OR 2.2, $95 \%$ CI 1.2-4.3, $p=0.014$ ) were found to be independent risk factors for RM involvement by the tumor in patients with T2-T4 Lauren diffuse/mixed pattern cancer.

When the analysis was restricted to only patients with gastric cancer, presence of lymphatic infiltration (OR 3.9, $95 \%$ CI 1.6-9.5, $p=0.002$ ), tumor diameter greater than $4 \mathrm{~cm}$ (OR 3.3, $95 \%$ CI 1.7-6.4, $p<0.001$ ), and serosa invasion (OR 2.3, $95 \%$ CI 1.2-4.6, $p=0.011$ ) were found to be the only independent risk factors associated with RM involvement.

\section{Discussion}

RM clearance is an issue of major importance in gastric cancer surgery. Depending on different case series, the prevalence of $\mathrm{R} 1$ resections ranges from 2.8 to $20 \%$ of operated-on patients [2]. The prognostic impact of this RM 
Table 4 Univariate and multivariate analysis of potential risk factors for $\mathrm{T} 2-\mathrm{T} 4$ Lauren intestinal pattern cancers between patients with a positive proximal or distal resection margin $(R M)$ (group A) or a negative proximal or distal $\mathrm{RM}$ (group B)

\begin{tabular}{|c|c|c|c|c|c|c|}
\hline Variable & Group A (45 patients). & Group B (229 patients) & $p^{\mathrm{a}}$ & OR & $95 \% \mathrm{CI}$ & $p^{\mathrm{a}}$ \\
\hline Age & $67.2 \pm 13.0$ years & $69.1 \pm 10.8$ years & 0.306 & & & \\
\hline \multicolumn{7}{|l|}{ Sex } \\
\hline Male & $72 \%$ & $61 \%$ & 0.215 & & & \\
\hline Female & $28 \%$ & $39 \%$ & & & & \\
\hline BMI & $24.9 \pm 3.7 \mathrm{~kg} / \mathrm{m}^{2}$ & $25.6 \pm 4.3 \mathrm{~kg} / \mathrm{m}^{2}$ & 0.610 & & & \\
\hline \multicolumn{7}{|l|}{ Type of surgery } \\
\hline Elective & $97 \%$ & $95 \%$ & 0.695 & & & \\
\hline Emergent & $3 \%$ & $5 \%$ & & & & \\
\hline \multicolumn{7}{|c|}{ Multivisceral resections } \\
\hline No & $82 \%$ & $98 \%$ & $<0.001$ & 2.5 & $0.4-16.6$ & 0.372 \\
\hline Yes & $18 \%$ & $2 \%$ & & & & \\
\hline \multicolumn{7}{|l|}{ NACT } \\
\hline No & $89 \%$ & $90 \%$ & 0.797 & & & \\
\hline Yes & $11 \%$ & $10 \%$ & & & & \\
\hline \multicolumn{7}{|c|}{ RM distance from tumor } \\
\hline$<3 \mathrm{~cm}$ & $91 \%$ & $63 \%$ & 0.001 & 4.0 & $1.1-14.5$ & 0.03 \\
\hline$\geq 3 \mathrm{~cm}$ & $9 \%$ & $37 \%$ & & & & \\
\hline \multicolumn{7}{|l|}{ EGJ location } \\
\hline No & $64 \%$ & $80 \%$ & 0.030 & 4.1 & $1.7-10.0$ & 0.002 \\
\hline Yes & $36 \%$ & $20 \%$ & & & & \\
\hline Tumor diameter & $5.2 \pm 2.2 \mathrm{~cm}$ & $4.7 \pm 2.5 \mathrm{~cm}$ & 0.214 & & & \\
\hline \multicolumn{7}{|l|}{ Tumor grade } \\
\hline G1-G2 & $34 \%$ & $51 \%$ & 0.048 & 1.2 & $0.5-2.8$ & 0.693 \\
\hline G3-G4 & $66 \%$ & $49 \%$ & & & & \\
\hline \multicolumn{7}{|c|}{ Signet ring cell histological appearance } \\
\hline No & $78 \%$ & $87 \%$ & 0.127 & & & \\
\hline Yes & $22 \%$ & $13 \%$ & & & & \\
\hline \multicolumn{7}{|c|}{ Multifocal disease } \\
\hline No & $95 \%$ & $99 \%$ & 0.173 & & & \\
\hline Yes & $5 \%$ & $1 \%$ & & & & \\
\hline \multicolumn{7}{|l|}{ Tumor invasion } \\
\hline No & $76 \%$ & $90 \%$ & 0.024 & 2.3 & $0.8-6.9$ & 0.129 \\
\hline Yes & $24 \%$ & $10 \%$ & & & & \\
\hline \multicolumn{7}{|c|}{ Vascular infiltration } \\
\hline No & $67 \%$ & $84 \%$ & 0.018 & 0.4 & $0.1-1.5$ & 0.526 \\
\hline Yes & $33 \%$ & $16 \%$ & & & & \\
\hline \multicolumn{7}{|c|}{ Lymphatic infiltration } \\
\hline No & $72 \%$ & $90 \%$ & 0.002 & 1 & $0.2-4.9$ & 0.775 \\
\hline Yes & $28 \%$ & $10 \%$ & & & & \\
\hline \multicolumn{7}{|c|}{ Perineural infiltration } \\
\hline No & $85 \%$ & $93 \%$ & 0.106 & & & \\
\hline Yes & $15 \%$ & $7 \%$ & & & & \\
\hline \multicolumn{7}{|c|}{ Lymph node status } \\
\hline N0 & $20 \%$ & $32 \%$ & 0.113 & & & \\
\hline $\mathrm{N}+$ & $80 \%$ & $68 \%$ & & & & \\
\hline \multicolumn{7}{|l|}{ Serosa invasion } \\
\hline No & $47 \%$ & $84 \%$ & $<0.001$ & 6.0 & $2.5-14.8$ & $<0.001$ \\
\hline Yes & $53 \%$ & $16 \%$ & & & & \\
\hline
\end{tabular}


Table 4 continued

\begin{tabular}{|c|c|c|c|c|c|c|}
\hline Variable & Group A (45 patients). & Group B (229 patients) & $p^{\mathrm{a}}$ & OR & $95 \% \mathrm{CI}$ & $p^{\mathrm{a}}$ \\
\hline \multicolumn{7}{|c|}{ HER2 gene overexpression/amplification } \\
\hline No & $96 \%$ & $96 \%$ & 1.000 & & & \\
\hline Yes & $4 \%$ & $4 \%$ & & & & \\
\hline \multicolumn{7}{|c|}{ TRG (only for NACT) } \\
\hline $1-3$ & $60 \%$ & $41 \%$ & 0.628 & & & \\
\hline $4-5$ & $40 \%$ & $59 \%$ & & & & \\
\hline
\end{tabular}

$C I$ confidence interval, EGJ esophagogastric junction, HER2 human epidermal growth factor receptor 2, $N A C T$ neoadjuvant chemotherapy, $O R$ odds ratio, TRG tumor regression grade

${ }^{a}$ Values in italic are significant

involvement by the tumor has been widely studied by several authors: in a multicenter Italian study, Morgagni et al. [4] showed that RM infiltration is an independent unfavorable factor in patients who underwent gastrectomy (OR 1.5), with a 5-year OS rate of $66 \%$ in R0 resections and $29 \%$ in R1 resections. Eastern authors [3, 5, 14, 15] observed similar results, with an unstratified 5-year survival rate ranging from 53 to $60 \%$ in the case of $\mathrm{R} 0$ resections, and from 13 to $26 \%$ in case of $\mathrm{R} 1$ resections. In the present study we found that the 5-year survival rate of the whole cohort was $59 \%$ in patients with negative RMs and $22 \%$ in patients with positive RMs; therefore, the data we are presenting perfectly overlap with those reported in the literature.

However, although the effect of $\mathrm{R} 1$ resection is undoubtedly negative on the whole series population, the definitive prognostic significance in a specific subgroup of patients is still to be defined. Sun et al. [3] found that R1 resections affect prognosis only in T1-T2 cancer patients, whereas Cho et al. [2] identified a worse survival only in N0-N1 cancer patients. These authors suggest that R1 resections may have a negative impact only in low-stage cancer patients, because the adverse effects of positive RMs might be overwhelmed by the more detrimental impact of the depth of invasion or nodal metastasis. In contrast, Morgagni [1] and Nakamura et al. [19] showed that the prognosis of patients with EGC is not affected by the presence of positive RMs, because of the excellent survival for this type of disease. Finally, in contrast to the above results, Wang et al. [14] found that positive margins affect survival despite the tumor category.

Pattern of recurrence analysis after R1 resection showed conflicting results, and may explain the aforementioned finding. Sun et al. [3] found that locoregional relapse in patients with positive RMs was more frequent than distant or peritoneal relapse, and that it was more frequent than in negative RM patients, as expected. In contrast, Wang et al. [14] and Bickenbach et al. [20] showed that the main recurrence pattern in $\mathrm{R} 1$ resection patients was peritoneal rather than locoregional. This finding may indicate that positive RM patients generally have a more advanced and more aggressive disease [20], whose systemic impact can mask the still present locoregional effects. In addition, in our study we found that patients with positive RMs had clinicopathological features significantly more advanced and more aggressive than those of $\mathrm{R} 0$ resection patients, especially concerning $\mathrm{T}$ category, $\mathrm{N}$ category, grading, and Lauren pattern. Such a pronounced difference between the two groups of patients can then explain the equally pronounced difference in survival as observed in this study as well as in previous ones.

Despite the fact that the real prognostic impact of positive RMs is still a matter of discussion, most authors [1, 2, $4,21,22]$ suggest that, in the case of $\mathrm{R} 1$ resection, a surgical retreatment of the patient aiming to achieve radical resection (if possible) should always be considered, in order to ensure optimal prognostic results. Lee et al. [23] showed that the OS rate and the recurrence rate are not changed if $\mathrm{R} 0$ resection is achieved by performing additional resection for positive RMs. Other authors, by contrast, found that surgical re-excision of infiltrated margin has a prognostic benefit only when disease extension is limited, as in case of limited nodal involvement [20, 24] or nontrasmural disease [20], and should therefore be attempted only in such cases.

Because it was shown that, in the case of $\mathrm{R} 0$ resection, the distance of free margin from the tumor does not impact prognosis [25, 26], several authors [6-9] and the Japanese Gastric Cancer Association [10] sought to determine what is the optimal distance of free margin from the tumor in order to reduce the risk of RM infiltration. However, findings have been heterogeneous so far.

IFS analysis has been reported to have excellent sensitivity, specificity, and accuracy [11, 27] in identifying infiltrated margins. Also in the present series, the positive prognostic value and negative prognostic value were $100 \%$ : all cases of positive margins on IFS analysis were confirmed by DHE, whereas no cases of $\mathrm{R} 1$ resections with negative RMs on IFS analysis were reported. Therefore, the use of IFS analysis to determine the extent of resection is 
Table 5 Univariate and multivariate analysis of potential risk factors for $\mathrm{T} 2-\mathrm{T} 4$ Lauren diffuse/mixed pattern cancers between patients with a positive proximal or distal resection margin $(R M)$ (group A) or a negative proximal or distal RM (group B)

\begin{tabular}{|c|c|c|c|c|c|c|}
\hline Variable & Group A (89 patients). & Group B (136 patients) & $p^{\mathrm{a}}$ & OR & $95 \% \mathrm{CI}$ & $p^{\mathrm{a}}$ \\
\hline Age & $63.5 \pm 15.1$ years & $65.3 \pm 13.6$ years & 0.368 & & & \\
\hline \multicolumn{7}{|l|}{ Sex } \\
\hline Male & $47 \%$ & $43 \%$ & 0.328 & & & \\
\hline Female & $53 \%$ & $57 \%$ & & & & \\
\hline BMI & $24.7 \pm 3.9 \mathrm{~kg} / \mathrm{m}^{2}$ & $23.8 \pm 4.2 \mathrm{~kg} / \mathrm{m}^{2}$ & 0.501 & & & \\
\hline \multicolumn{7}{|l|}{ Type of surgery } \\
\hline Elective & $90 \%$ & $94 \%$ & 0.412 & & & \\
\hline Emergent & $10 \%$ & $6 \%$ & & & & \\
\hline \multicolumn{7}{|c|}{ Multivisceral resections } \\
\hline No & $88 \%$ & $95 \%$ & 0.076 & & & \\
\hline Yes & $12 \%$ & $5 \%$ & & & & \\
\hline \multicolumn{7}{|l|}{ NACT } \\
\hline No & $91 \%$ & $87 \%$ & 0.397 & & & \\
\hline Yes & $9 \%$ & $13 \%$ & & & & \\
\hline \multicolumn{7}{|c|}{$\mathrm{RM}$ distance from tumor } \\
\hline$<5 \mathrm{~cm}$ & $90 \%$ & $88 \%$ & 0.808 & & & \\
\hline$\geq 5 \mathrm{~cm}$ & $10 \%$ & $12 \%$ & & & & \\
\hline \multicolumn{7}{|l|}{ EGJ location } \\
\hline No & $77 \%$ & $92 \%$ & 0.003 & 2.8 & $1.1-7.0$ & 0.033 \\
\hline Yes & $23 \%$ & $8 \%$ & & & & \\
\hline Tumor diameter & $6.1 \pm 3.7 \mathrm{~cm}$ & $4.1 \pm 2.1 \mathrm{~cm}$ & $<0.001$ & & & \\
\hline$\leq 4 \mathrm{~cm}$ & $28 \%$ & $64 \%$ & $<0.001$ & 3.5 & $1.8-6.7$ & $<0.001$ \\
\hline$>4 \mathrm{~cm}$ & $72 \%$ & $36 \%$ & & & & \\
\hline \multicolumn{7}{|l|}{ Tumor grade } \\
\hline G1-G2 & $6 \%$ & $7 \%$ & 1.000 & & & \\
\hline G3-G4 & $94 \%$ & $93 \%$ & & & & \\
\hline \multicolumn{7}{|c|}{ Signet ring cell histological appearance } \\
\hline No & $25 \%$ & $33 \%$ & 0.323 & & & \\
\hline Yes & $75 \%$ & $67 \%$ & & & & \\
\hline \multicolumn{7}{|c|}{ Multifocal disease } \\
\hline No & $99 \%$ & $98 \%$ & 1.000 & & & \\
\hline Yes & $1 \%$ & $2 \%$ & & & & \\
\hline \multicolumn{7}{|l|}{ Tumor invasion } \\
\hline No & $74 \%$ & $90 \%$ & 0.002 & 1.5 & $0.6-3.9$ & 0.36 \\
\hline Yes & $26 \%$ & $10 \%$ & & & & \\
\hline \multicolumn{7}{|c|}{ Vascular infiltration } \\
\hline No & $78 \%$ & $89 \%$ & 0.037 & 1 & $0.4-2.6$ & 0.906 \\
\hline Yes & $22 \%$ & $11 \%$ & & & & \\
\hline \multicolumn{7}{|c|}{ Lymphatic infiltration } \\
\hline No & $74 \%$ & $91 \%$ & 0.001 & 4.2 & $1.7-10.3$ & 0.002 \\
\hline Yes & $26 \%$ & $9 \%$ & & & & \\
\hline \multicolumn{7}{|c|}{ Perineural infiltration } \\
\hline No & $88 \%$ & $95 \%$ & 0.063 & & & \\
\hline Yes & $12 \%$ & $5 \%$ & & & & \\
\hline \multicolumn{7}{|c|}{ Lymph node status } \\
\hline N0 & $13 \%$ & $26 \%$ & 0.030 & 1.5 & $0.6-3.5$ & 0.671 \\
\hline $\mathrm{N}+$ & $87 \%$ & $74 \%$ & & & & \\
\hline \multicolumn{7}{|l|}{ Serosa invasion } \\
\hline No & $36 \%$ & $63 \%$ & $<0.001$ & 2.2 & $1.2-4.3$ & 0.014 \\
\hline Yes & $64 \%$ & $37 \%$ & & & & \\
\hline
\end{tabular}


Table 5 continued

\begin{tabular}{|c|c|c|c|c|c|c|}
\hline Variable & Group A (89 patients). & Group B (136 patients) & $p^{\mathrm{a}}$ & OR & $95 \% \mathrm{CI}$ & $p^{\mathrm{a}}$ \\
\hline \multicolumn{7}{|c|}{ HER2 gene overexpression/amplification } \\
\hline No & $99 \%$ & $99 \%$ & 1.000 & & & \\
\hline Yes & $1 \%$ & $1 \%$ & & & & \\
\hline \multicolumn{7}{|c|}{ TRG (only for NACT) } \\
\hline $1-3$ & $67 \%$ & $19 \%$ & 0.155 & & & \\
\hline $4-5$ & $33 \%$ & $81 \%$ & & & & \\
\hline
\end{tabular}

CI confidence interval, EGJ esophagogastric junction, HER2 human epidermal growth factor receptor 2, $N A C T$ neoadjuvant chemotherapy, $O R$ odds ratio, TRG tumor regression grade

${ }^{a}$ Values in italic are significant

the safest way to reduce the risk or R1 margins and the subsequent problem of surgical re-excision, but it is not common practice and there is no agreement as to in which patients it should be performed [12].

Firstly, the usefulness of performing IFS analysis should also be related to the site of the margin examined (proximal or distal) and the type of resection (subtotal or total gastrectomy). IFS analysis makes sense if there is the technical possibility to perform an extent of resection in the case of a positive margin. A distal margin, both in total and in subtotal gastrectomy, can be analyzed if the duodenal section is performed just beyond the pylorus. In the case of a positive margin it is generally possible to prepare the first part of the duodenum to further extent the resection. In contrast, if no further duodenal resection is possible, IFS analysis on the distal margin should be performed only on those highly selected cases where pancreaticoduodenectomy can be performed. The proximal marginal in subtotal gastrectomy does not present particular issues, because further gastric resection or conversion to total gastrectomy can always be performed. In contrast, in the case of total gastrectomy, it is useful to analyze the proximal margin if an esophageal resection (even by thoracotomy) can be performed.

Moreover, IFS analysis is extremely time-consuming and resource-consuming (the estimated cost for a patient is about $\$ 1000$ for both RMs) [13], and its routine use in all gastric resections, as suggested by some authors $[14,15$, 23], does not seem reliable.

Therefore, IFS analysis should be selectively performed in those cases where the risk of infiltrated margins is high and there is the possibility to perform an extent of resection. For this reason, several studies have been performed to identify risk factors associated with RM involvement, and a systematic review of this topic has been published recently [28]. According to the different studies, T category $[2,3,14,20], \mathrm{N}$ category $[2,3,14,20]$, tumor size $[3,5,14$, 23], proximal tumor location [2, 23, 29], high tumor grading [2, 20], Bormann type IV gastric cancer [2, 23], Lauren diffuse pattern [20, 29], and signet ring cell histological appearance [23] have been identified as independent risk factors for RM involvement.

All these factors, together with several other variables, many of which have never been previously studied, were selectively analyzed in this study with a clinical-oriented method. To the best of our knowledge, this is the first study in which risk factor analysis has been performed stratifying patients according to $\mathrm{T}$ category and Lauren pattern, which have been identified by most authors and by the JGCA as the principal factors associated with RM involvement. This allowed us to perform an analysis aimed at providing the surgeon with a precise assessment of case-specific RM involvement risk, and, consequently, of the usefulness of performing IFS analysis.

Such an outcome was made possible because of our collecting one of the largest cohort of patients with RM involvement, including not only patients with $\mathrm{R} 1$ resection on DHE but also patients with positive IFS analysis results, who would actually have had a R1 resection if the IFS analysis had not been performed.

According to the results of the present study, in T1 cancer patients the only identified risk factor for RM involvement is a margin distance of less than $2 \mathrm{~cm}(\mathrm{OR}$ 15.7). This finding perfectly overlaps with the JGCA guidelines [10], which suggest keeping a distance of at least $2 \mathrm{~cm}$ between the tumor and the resection line to avoid the risk of margin infiltration. Thus, IFS analysis is indicated only if the resection cannot respect this safe distance. A limitation of this subgroup analysis is the small number of group A patients, due to the difficulty in finding EGC patients with RM involvement. Nevertheless, because, as previously discussed, the prognostic impact of $\mathrm{R} 1$ resection in EGC is still to be defined, the use of IFS analysis in these patients may be a questionable choice.

In T2-T4 Lauren intestinal pattern cancer patients, the 3-cm margin distance suggested by the JGCA was also found to be an independent risk factor for RM involvement (OR 4.0). However, in these patients, other important identified risk factors are EGJ tumor location (OR 4.1) and, above all, serosa invasion (OR 6.0). Because all these 


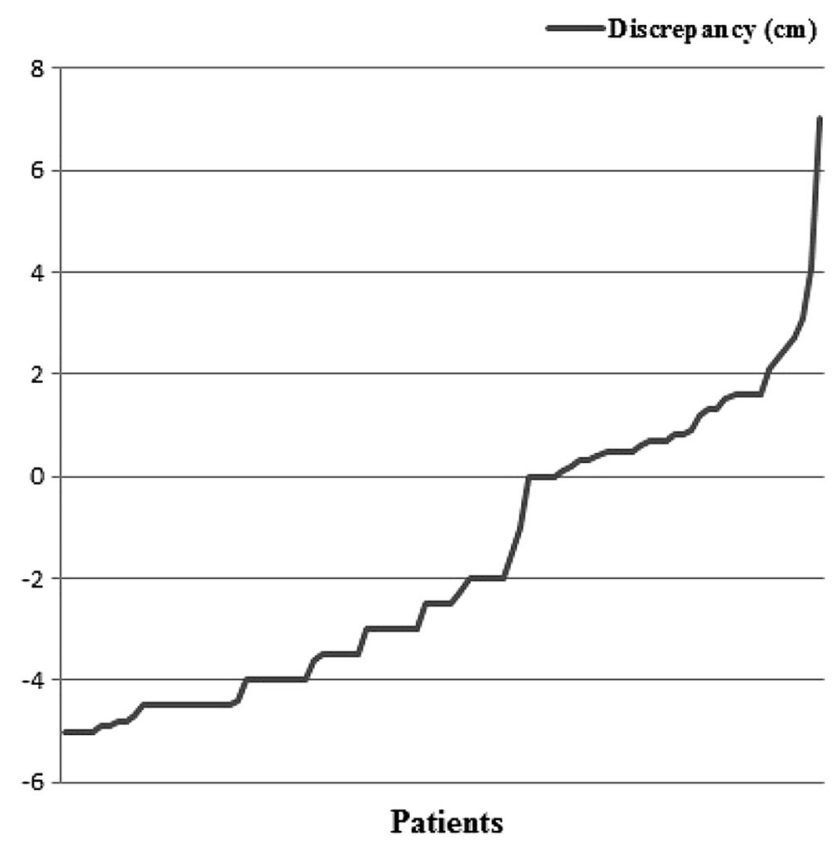

Fig. 2 Discrepancy between the resection margin and the 5-cm cutoff suggested by the Japanese Gastric Cancer Association in patients with T2-T4 Lauren diffuse/mixed pattern cancers with resection margin involvement

factors are known preoperatively and/or intraoperatively, IFS analysis use should therefore be mandatory in these selected cases.

Finally, also in T2-T4 Lauren diffuse/mixed pattern cancer patients, EGJ tumor location (OR 2.8) and serosa invasion (OR 2.2) have been shown to be independent risk factors, together with tumor size greater than $4 \mathrm{~cm}$ (OR 3.5) and the presence of lymphatic infiltration on DHE (OR 4.2). Unlike the previous subgroups, however, in this case the margin distance suggested by the JGCA guidelines is not associated with a higher risk of RM infiltration, considering both the entire group of patients and only those affected by gastric cancer. Although the mean distance between the tumor and the RM was only $3.3 \mathrm{~cm}$, we found RM infiltration from $5.1 \mathrm{~cm}$ up to even $12 \mathrm{~cm}$ away from the tumor (see Fig. 2). In such a scenario, defining a reliable safety margin does not seem to be an easy task. Moreover, the most relevant risk factor (presence of lymphatic infiltration) is a variable that can be known only postoperatively. For these reasons, and since Lauren diffuse/mixed cancer has the higher incidence of RM involvement, the use of IFS analysis should be widely applied in the case of T2-T4 diffuse pattern cancers, especially in advanced forms.

In conclusion, RM involvement has a negative prognostic impact in patients who underwent gastric surgery, and it is associated with more aggressive and more advanced clinicopathological features. The use of IFS analysis can reduce the rate of $\mathrm{R} 1$ resection and the need for surgical re-excision, but it should be selectively applied in patients with higher risk of RM involvement. Margin distance less than $2 \mathrm{~cm}$ is an independent risk factor for RM involvement in T1 cancer, serosa invasion, EGJ location, and margin distance less than $3 \mathrm{~cm}$ are independent risk factors for RM involvement in T2-T4 Lauren intestinal pattern cancer, and lymphatic infiltration, tumor size larger than $4 \mathrm{~cm}$, EGJ location, and serosa invasion are independent risk factors for RM involvement in T2-T4 Lauren diffuse/mixed pattern cancer. IFS analysis should be routinely performed in those patients with high risk of positive RMs, especially in Lauren diffuse pattern cancers.

\section{Compliance with ethical standards}

Conflict of interest The authors declare that they have no conflict of interest.

Human rights statement and informed consent All procedures followed were in accordance with the ethical standards of the responsible committee on human experimentation (institutional and national) and with the Helsinki Declaration of 1964 and later versions. Informed consent or substitute for it was obtained from all patients for their being included in the study.

\section{References}

1. Morgagni P, Garcea D, Marrelli D, de Manzoni G, Natalini G, Kurihara H, Marchet A, Vittimberga G, Saragoni L, Roviello F, Di Leo A, De Santis F, Panizza V, Nitti D. Does resection line involvement affect prognosis in early gastric cancer patients? An Italian multicentric study. World J Surg. 2006;30(4):585-9.

2. Cho BC, Jeung HC, Choi HJ, Rha SY, Hyung WJ, Cheong JH, Noh SH, Chung HC. Prognostic impact of resection margin involvement after extended (D2/D3) gastrectomy for advanced gastric cancer: a 15-year experience at a single institute. J Surg Oncol. 2007;95(6):461-8.

3. Sun Z, Li DM, Wang ZN, Huang BJ, Xu Y, Li K, Xu HM. Prognostic significance of microscopic positive margins for gastric cancer patients with potentially curative resection. Ann Surg Oncol. 2009;16(11):3028-37.

4. Morgagni P, Garcea D, Marrelli D, De Manzoni G, Natalini G, Kurihara H, Marchet A, Saragoni L, Scarpi E, Pedrazzani C, Di Leo A, De Santis F, Panizzo V, Nitti D, Roviello F. Resection line involvement after gastric cancer surgery: clinical outcome in nonsurgically retreated patients. World J Surg. 2008;32(12): 2661-7.

5. Nagata T, Ichikawa D, Komatsu S, Inoue K, Shiozaki A, Fujiwara H, Okamoto K, Sakakura C, Otsuji E. Prognostic impact of microscopic positive margin in gastric cancer patients. J Surg Oncol. 2011;104(6):592-7.

6. Bozzetti F, Bonfanti G, Bufalino R, Menotti V, Persano S, Andreola S, Doci R, Gennari L. Adequacy of margins of resection in gastrectomy for cancer. Ann Surg. 1982;196(6):685-90.

7. Ito H, Clancy TE, Osteen RT, Swanson RS, Bueno R, Sugarbaker DJ, Ashley SW, Zinner MJ, Whang EE. Adenocarcinoma of the gastric cardia: what is the optimal surgical approach? J Am Coll Surg. 2004;199(6):880-6.

8. Papachristou DN, Fortner JG. Local recurrence of gastric adenocarcinomas after gastrectomy. J Surg Oncol. 1981;18(1): 47-53. 
9. Kim JH, Park SS, Kim J, Boo YJ, Kim SJ, Mok YJ, Kim CS. Surgical outcomes for gastric cancer in the upper third of the stomach. World J Surg. 2006; 30(10):1870-6; discussion 1877-8.

10. Japanese Gastric Cancer Association. Japanese gastric cancer treatment guidelines 2010 (ver. 3). Gastric Cancer. 2011;14(2): 113-23.

11. Ferreiro JA, Myers JL, Bostwick DG. Accuracy of frozen section diagnosis in surgical pathology: review of a 1-year experience with 24,880 cases at Mayo Clinic Rochester. Mayo Clin Proc. 1995;70(12):1137-41.

12. Shen JG, Cheong JH, Hyung WJ, Kim J, Choi SH, Noh SH. Intraoperative frozen section margin evaluation in gastric cancer of the cardia surgery. Hepatogastroenterology. 2006;53(72): 976-8.

13. DiNardo LJ, Lin J, Karageorge LS, Powers CN. Accuracy, utility, and cost of frozen section margins in head and neck cancer surgery. Laryngoscope. 2000;110(10 Pt 1):1773-6.

14. Wang SY, Yeh CN, Lee HL, Liu YY, Chao TC, Hwang TL, Jan YY, Chen MF. Clinical impact of positive surgical margin status on gastric cancer patients undergoing gastrectomy. Ann Surg Oncol. 2009;16(10):2738-43.

15. Woo JW, Ryu KW, Park JY, Eom BW, Kim MJ, Yoon HM, Park SR, Kook MC, Choi IJ, Kim YW, Park YI. Prognostic impact of microscopic tumor involved resection margin in advanced gastric cancer patients after gastric resection. World J Surg. 2014;38(2): 439-46.

16. Peters SR. A practical guide to frozen section technique; New York: Springer Science+Business Media; 2010.

17. Rowinsky EK. The erbB family: targets for therapeutic development against cancer and therapeutic strategies using monoclonal antibodies and tyrosine kinase inhibitors. Annu Rev Med. 2004;55:433-57.

18. Mandard AM, Dalibard F, Mandard JC, Marnay J, Henry-Amar M, Petiot JF, Roussel A, Jacob JH, Segol P, Samama G, et al. Pathologic assessment of tumor regression after preoperative chemoradiotherapy of esophageal carcinoma. Clinicopathologic correlations. Cancer. 1994;73(11):2680-6.

19. Nakamura K, Ueyama T, Yao T, Xuan ZX, Ambe K, Adachi Y, Yakeishi Y, Matsukuma A, Enjoji M. Pathology and prognosis of gastric carcinoma. Findings in 10,000 patients who underwent primary gastrectomy. Cancer. 1992;70(5):1030-7.

20. Bickenbach KA, Gonen M, Strong V, Brennan MF, Coit DG. Association of positive transection margins with gastric cancer survival and local recurrence. Ann Surg Oncol. 2013;20(8): 2663-8.

21. Songun I, Bonenkamp JJ, Hermans J, van Krieken JH, van de Velde CJ. Prognostic value of resection-line involvement in patients undergoing curative resections for gastric cancer. Eur $\mathrm{J}$ Cancer. 1996;32A(3):433-7.

22. Cascinu S, Giordani P, Catalano V, Agostinelli R, Catalano G. Resection-line involvement in gastric cancer patients undergoing curative resections: implications for clinical management. Jpn J Clin Oncol. 1999;29(6):291-3.

23. Lee JH, Ahn SH, Parkdo J, Kim HH, Lee HJ, Yang HK. Clinical impact of tumor infiltration at the transected surgical margin during gastric cancer surgery. J Surg Oncol. 2012;106(6):772-6.

24. Kim SH, Karpeh MS, Klimstra DS, Leung D, Brennan MF. Effect of microscopic resection line disease on gastric cancer survival. J Gastrointest Surg. 1999;3(1):24-33.

25. Jang YJ, Park MS, Kim JH, Park SS, Park SH, Kim SJ, Kim CS, Mok YJ. Advanced gastric cancer in the middle one-third of the stomach: should surgeons perform total gastrectomy? J Surg Oncol. 2010;101(6):451-6.

26. Lee JH, Kim YI. Which is the optimal extent of resection in middle third gastric cancer between total gastrectomy and subtotal gastrectomy? J Gastric Cancer. 2010;10(4):226-33.

27. Torp SH, Skjørten FJ. The reliability of frozen section diagnosis. Acta Chir Scand. 1990;156(2):127-30.

28. Raziee HR, Cardoso R, Seevaratnam R, Mahar A, Helyer L, Law C, Coburn N. Systematic review of the predictors of positive margins in gastric cancer surgery and the effect on survival. Gastric Cancer. 2012;15(Suppl 1):S116-24.

29. Kim SY, Hwang YS, Sohn TS, Oh SJ, Choi MG, Noh JH, Bae $\mathrm{JM}$, Kim S. The predictors and clinical impact of positive resection margins on frozen section in gastric cancer surgery. J Gastric Cancer. 2012;12(2):113-9. 